\title{
Reflexive anaphors and association with focus*
}

\author{
Giorgos Spathas \\ University of Stuttgart
}

\begin{abstract}
This paper investigates the behavior of the reflexive anaphor herself in English in conditions of narrow focus and argues in favor of (i) a theory of focus that is based around the notion of Current Question, and (ii) a theory of association with focus that assumes that some, but not all, focus associating operators exhibit conventional association; i.e. some, but not all, focus associating operators have lexical semantics that directly encode association with focus. The argument is based on the independently motivated observation that there exist asymmetries with regard to disjoint reference effects between subject and object wh-questions.
\end{abstract}

Keywords: association with focus, Question-Answer congruence, reflexive anaphors

\section{Introduction}

As is well-known, the placement of accent in an utterance can have truthconditional effects in the presence of certain semantic operators, a phenomenon called association with focus. For example, in the case of the exclusive operator only, (1a), with focus on Bill, entails that John introduced no one else to Sue except Bill, whereas (1b), with focus on Sue, entails that John introduced Bill to no one but Sue. ${ }^{1,2}$

\footnotetext{
* I wish to thank Artemis Alexiadou, Florian Schäfer, Arndt Riester, and Michael Wagner for discussion. All errors are my own. Many thanks go to Bert Le Bruyn for his help with some formatting issues.

${ }^{1}$ I assume that the syntactic correlate of focus is F-marking (cf. Jackendoff 1972) and syntactic nodes can be freely annotated with a privative feature $\mathrm{F}$ (ocus). In the phonological component, Fmarking is linked to prosodic prominence relative to a domain of focus (cf. Truckenbrodt 1995). I make the simplifying assumption that the domain of focus is always the full sentence. Prominence is indicated here with small capitals. Prosodic prominence in English correlates perceptually with pitch accent, which is acoustically realized with a local maximum or minimum of the fundamental frequency.

${ }^{2}$ For the purposes of this paper, F-marking on a word corresponds to F-marking on that word alone. If required, F-marking of wider constituents will be indicated with the use of angled brackets.
} 
(1) a. John introduced BILLF to Sue.

b. John introduced Bill to SUEF.

The phenomenon of association with focus has received significant attention in the semantic literature and several frameworks have been developed to explain this type of meaning sensitivity to prosody (most prominently, Alternative Semantics, as in Rooth 1985, 1992, and Structured Meanings, as in von Stechow 1990, Krifka 1992). In this paper I assume the framework of Alternative Semantics, so that each expression is associated with a set of alternatives, its Focus Semantic Value (FSV), and I focus on the extent to which the meaning of utterances with focus associating operators (FAOs) is determined by the lexical meanings of FAOs. In this respect, Rooth (1992) distinguishes between three possible theories of association with focus: (i) weak theories, according to which the link between FAOs and foci is part of the conventionalized lexical meaning of the operators (Rooth 1985), (ii) strong theories, according to which focus sensitivity emerges from the workings of general pragmatic mechanisms (Rooth 1992, von Fintel 1994, Roberts 1996, 2004), and (iii) intermediate theories, according to which conventional association is true of some but not all FAOs (Rooth 1992, Beaver and Clark 2008). Roberts 1996, 2004 argues in favor of a strong theory built on a question-based discourse model. Beaver and Clark 2008 undergoes a comprehensive comparison between different FAOs and concludes in favor of an intermediate theory cast in (a version of) Roberts' question-based model. This paper argues in favor of the position in Beaver and Clark 2008, i.e. an intermediate theory of association with focus that is built on a question-based discourse model.

My argument is based on the distribution of the alternatives generated by narrow focus on reflexive anaphors in English. Spathas (2010, 2011) observes that narrow focus on a reflexive anaphor is licensed in two rather different environments (see also Ahn 2012). For example, in the Question-Answer (QA) pairs in (2) and (3), the same sentence with the same intonational contour is used to answer both an object $w h$-question as in (2), as well as a subject $w h$-question as in (3). The regular meaning of $(2 / 3 \mathrm{~A})$ is, of course, the same in both cases (roughly, the proposition that Zelda praised Zelda), but under focus-sensitive rules of QA-Congruence the generated alternatives should be able to vary both in the object position (Zelda praised $x$ ) for (2) to be felicitous, and in the subject position ( $x$ praised Zelda) for (3) to be felicitous. We refer to alternatives of the form Zelda praised $x$ as Object Alternatives (OA) and to alternatives of the form $x$ praised Zelda as Subject Alternatives (SA). ${ }^{3}$

\footnotetext{
${ }^{3}$ Notice that prosodic prominence of a reflexive anaphor in argument position unambiguously indicates narrow focus on the anaphor; in the case of wider focus domains, as in, e.g., VP focus in
} 
Reflexive anaphors and association with focus

(2) Q: Who did Zelda praise?

A: She praised herSELFF.

(3) Q: Who praised Zelda?

A: ? She praised herSELFF.

As Spathas $(2010,2011)$ argues, the existence of SA forces a treatment of reflexive anaphors in terms of reflexivizing functions (as done mostly in the Categorial Grammar literature), as in (4a), rather than designated bound variables. Assuming (4a), a focused reflexive anaphor contrasts with other argument reducing operations like passivization and anti-passivization. The FSV of (2/3A), then, includes both SA and OA, as in, e.g., (4b).

(4) a. [[herself]] $=\lambda R_{\text {eet }} \lambda x$. $R(x)(x)$

b. \{Zelda praised Zelda, Oscar praised Zelda, Zelda praised Oscar, ... \}

This paper assumes this result and investigates further the distribution of SA under different focus associating operators. It is shown that (i) SA are generally less accessible than OA, and (ii) SA do not arise with conventionally associating operators, i.e. operators that make direct reference to alternatives in their lexical semantics (in a way to be made precise below). These two generalizations are argued to receive straightforward explanations if (i) focus theory is built based on a question-based model, and (ii) conventional association with focus is association with the Current Question. The account is based on the additional, independently motivated observation that there exist asymmetries with regard to the presence of disjoint reference effects between object and subject whquestions.

The paper is organized as follows. Section two establishes the two major generalizations covering the distribution of Subject and Object Alternatives. Section three presents an asymmetry between subject and object wh-questions; only the former exhibit a disjoint reference effect. Section four offers some new evidence in favor of a Current Question-based theory of focus and explains the reduced accessibility of SA on the basis of such a theory. Section five explains the impossibility of SA under conventionally associating operators under the assumption that conventional association is association with the Current Question. Section six concludes.

(i), the reflexive anaphor necessarily prosodically subordinates to the verb. See Spathas 2010, Ahn 2012 for alternative explanations of this pattern.

(i) Q: What did Zelda do?

A: Zelda [PRAISED herself] $]_{\mathrm{F}}$. 


\section{The distribution of subject and object alternatives}

This section establishes the two major generalizations that govern the behavior of SA. It is shown that SA, when available, is less accessible than OA. Moreover, $\mathrm{SA}$ is possible in free focus environments and in cases of quasi- and freeassociation, but not in cases of conventional association with focus. I proceed by first considering cases of free focus, before moving to cases of association with focus.

\subsection{Free focus}

The distribution of F-features in cases of free focus is determined by informationstructural considerations, usually some characterization of the notion of oldinformation (see particularly Schwarzschild 1999). For current purposes I assume the notion of Match in (5) (from Büring 2008). ${ }^{4,5}$ Assuming a Kartunnen/ Hamblin style semantics for questions, according to which they denote sets of possible answers, in the case of QA-pairs Match is usually taken to require that the meaning of the question is a subset of the answer's FSV, as in (6) (from Beaver and Clark 2008). ${ }^{6}$

\section{(5) Match}

There is some element $\mathrm{M}$ in the Focus Semantic Value of a sentence $\alpha$ which is a salient meaning in the discourse.

(6) Focus Principle

[Q] is a subset of the Focus Semantic Value of some part of A.

The distribution of OA and SA in cases of free focus is subject to (7). According to (7) there exists an asymmetry between OA and SA. This can be seen in the QA-pairs given in (2) and (3). As we observed there, both OA and SA are possible. However, in the case of a subject wh-question, as in (3), the accent

\footnotetext{
${ }^{4}$ As in the phonological component, I make the simplifying assumption that only sentences are domains of focus (i.e. Match is checked at the sentential level). I also do not discuss the necessary Economy component of focus theory, which is not relevant for the data discussed here. For discussion and different approaches to the formation of focus domains (and their relation with Economy) see Schwarzschild 1999, Wagner 2005, Büring 2008, Spathas 2010, among others.

5 Rooth (1992) treats the requirement posed by Match as a presupposition of his focus interpretation operator, the squiggle.

${ }^{6}$ The Focus Principle is weaker that Match in that it is satisfied if some part of the answer satisfies the relevant requirement. This assumption simplifies the analysis of FAOs (see Beaver and Clark 2008: 37 for discussion). Ideally, it should follow from a theory of focus domains. I do not have the space to go into this issue here.
} 
pattern is somewhat degraded. ${ }^{7}$ The same pattern is observed in the corrective contexts in (8) and (9).

(7) Generalization One

Everything else being equal, Object Alternatives are more accessible than Subject Alternatives.

(8) A: Zelda praised Oscar.

B: No, she praised herSELFF.

(9) A: Oscar praised Zelda.

B: ? No, she praised herSELFF.

\subsection{Association with focus}

In their typology of FAOs, Beaver and Clark (2008) distinguish between three classes of FAOs: Quasi-associating Operators (QAOs), Free-associating Operators (FrAOs), and Conventionally-associating Operators (CAOs). We present here the basic facts about SA following this typology.

\subsubsection{Quasi-associating operators}

QAOs are non-veridical, propositional operators like negation, intentional propositional modifiers, belief operators, etc. Consider, e.g., negation in (10) and (11). Although the effects of prosody are, in this case, not truth-conditional, differences in accent placement do lead to different inferences. Narrow focus on herself can generate two inferences: (i) that Zelda praised someone other than Zelda (OA), and (ii) that someone other than Zelda praised Zelda (SA). The availability of the two inferences is checked with two possible continuations, one compatible with OS (She praised Oscar in (10)) and one compatible with SA (Oscar praised her in (11)). The effects of (7) are manifested in such cases, as well. When confronted with (12) and no continuation, speakers always understand (12) to generate an inference based on OA, not SA. Similarly, (11), although perfectly possible, does seem to be somewhat degraded when compared to (10). Typically, speakers tend to warm-up to examples like (11) as the possibility of SA becomes more prominent/activated. I notate this state of affairs by adding a question mark in front of continuations licensed by SA.

(10) Zelda didn't praise herSELFF. She praised Oscar. $O A$

(11) Zelda didn't praise herSELFF. ? Oscar praised her.

(12) Zelda didn't praise herSELFF.

\footnotetext{
${ }^{7}$ Some speakers offer alternatives like the double focus variant ZELDA praised herSELF. See Spathas 2010, 2011 for discussion of this and other alternatives.
} 
Negation forms a good environment for SA to arise in, especially in examples with non-animate subjects, since SA in these cases generates the only plausible inferences. ${ }^{8}$ In (13), for example, the only plausible inference is that someone other than the room should go clean the room (SA), not, of course, that the room should clean something other than itself $(\mathrm{OA})$. In these cases, SA is perfectly natural.

(13) Go clean your room - it won't clean itSELFF.

Other QAOs like the intentional modifier perhaps in (14) and (15) and the belief operator think in (16) and (17) behave similarly.

(14) Perhaps Zelda praised herSELFF. Or perhaps she praised Oscar. $O A$

(15) Perhaps Zelda praised herSELFF. ? Or perhaps Oscar praised her. $S A$

(16) I think Zelda praised herSELFF. She didn't praise anyone else. $O A$

(17) I think Zelda praised herSELFF. ? No one else praised her. $S A$

\subsubsection{Free-associating operators (FrAOs)}

Free-associating operators quantify over an implicit domain, semantically represented as a variable over propositions. FrAOs include quantificational adverbs (18)/(19), generics/modals (20)/(21), and superlatives $(22) /(23)$, among others. As with QAOs, SA are possible but less accessible than OAs.

(18) Zelda always praises herSELFF.

She never praises other people.

(19) Zelda always praises herSELFF.

? No one else ever praises her.

(20) Ambitious people must praise themSELVES $\mathrm{F}$.

They shouldn't praise other people.

$O A$

(21) Lawyers must defend themSELVES ${ }_{\mathrm{F}}$.

? They are not allowed to have other lawyers defend them.

(22) Zelda praised herSELFF the longest.

She only mentioned Oscar in passing.

$O A$

\footnotetext{
${ }^{8}$ Stephens 2006 discusses examples like These jokes write themSELVES $F$ under the name 'virtual reflexive construction'. She claims that such cases exhibit an independent argument structure configuration. According to Stephens, virtual reflexives 'convey that the given activity is particularly easy for some agent to accomplish' (Stephens 2006: 277), while also 'allowing a partial transfer of agentivity to the patient-subject' (Stephens 2006: 143). The examples can be subsumed under the present proposal; they exhibit nothing more than regular SA readings of focused reflexivizers that associate with a covert FAO, probably Krifka's (1995) Emphatic Focus operator that indicates that the asserted proposition is particularly unlikely with respect to its alternatives.
} 
Reflexive anaphors and association with focus

(23) Zelda praised herSELFF the longest.

? Oscar only mentioned her in passing.

\subsubsection{Conventionally-associating operators (CAOs)}

Conventionally Associating Operators are also operators that have been assumed to include implicit variables. The resolution of the implicit variable, however, is not done through pragmatic mechanisms, but is encoded in the conventional lexical meanings of the operators. Beaver and Clark (2008) identify exclusives (24)/(25), scalar additives (26)/(27), additives (28)/(29), and particularizers (30)/(31) as CAOs. For all these, SA are not possible. The contrast between CAOs and other associating operators leads to the generalization in (32). ${ }^{9,10}$

(24) Zelda only praised herSELFF. She didn't praised anyone else.

$O A$

(25) Zelda only praised herSELFF. \# No one else praised her. $S A$

(26) Zelda praised many people. She even praised herSELFF. $O A$

(27) Many people praised Zelda.\# She even praised herSELFF. $S A$

(28) First, Zelda praised Oscar. Later, she praised herSELFF too. $O A$

(29) First, Oscar praised Zelda. \# Later, she praised herSELFF too. $S A$

(30) Zelda praised many people. E.g., she praised herSELFF. $O A$

(31) Many people praised Zelda.\# E.g, she praised herSELFF. $S A$

(32) Generalization Two

Object Alternatives are possible with all Focus Associating Operators.

Subject Alternatives are possible with Quasi- and Free-associating

Operators, but not with Conventionally-associating Operators.

\subsection{Always vs. only}

Beaver and Clark (2008) base their distinction between FrAOs and CAOs on a detailed comparison between the operators always and only. We can directly compare the behavior of only and always with regard to the licensing of SA in cases of quantification over events. ${ }^{11}$ Consider the minimal contrast in (33). The

\footnotetext{
${ }^{9}$ Some individual variation among speakers exists; speakers might find some CAOs better with SA than others. Importantly, no CAO is consistently judged on a par with QAOs and FrAOs, and all CAOs are consistently reported to be worse than QAOs and FrAOs.

${ }^{10}$ For most speakers, SA consistently arise with a certain class of predicates, that of Naturally Reflexive Verbs, i.e. verbs that have intransitive reflexive variants like wash, dress, etc. Spathas (2012) argues that in such cases herself is not an anaphor in argumental position but rather an adverbial anti-assistive intensifier, as in Mary built the house herself.

${ }^{11}$ For event-based meanings of only see, e.g., Bonomi and Casalegno 1993, Beaver and Clark 2008: 155-6.
} 
context in (33) forces a SA reading of (33a) and (33b), but only the variant with always is felicitous. Notice that the contrast is specific to SA; OA is possible for both only and always, as shown in (34).

(33) Context: Oscar is a petty criminal who always finds himself having to face some charge in court. Being a lawyer himself, he does not need to hire another attorney to defend him; if he chooses, he can defend himself in court. Does he ever use this option?

a. ? Yes, he always defends himSELFF (when accused of something).

b. \#Yes, he only defends himseLFF (when accused of something).

'Whenever someone defends Oscar, Oscar defends Oscar.'

(34) Context: Oscar is a petty criminal who usually works with his brother. They always find themselves having to face some charge or other in court. Being a lawyer himself, Oscar does not need to hire another attorney to defend him; if he chooses, he can defend himself in court. In fact, if he wants to, he can defend his brother. Does he ever do that?

a. ? No, he always defends himSELFF.

b. ? No, he only defends himSELFF.

'Whenever Oscar defends someone, Oscar defends Oscar.'

\section{Subject-object asymmetries in disjoint reference effects}

The analysis of the two generalizations above relies on an independent observation: subject $w h$-questions exhibit a disjoint reference effect, whereas object wh-questions do not. Consider first the QA-pair in (35). Speaker A answers the object $w h$-question by first asserting that Zelda praised no one and then continuing with the assertion that Zelda praised Zelda, an illegitimate move. The infelicity of She praised herSELF in (35A) can be explained as follows. By the use of She praised no one, the speaker asserts that all the propositions in [[Whom did Zelda praise]] are false. This includes the proposition that Zelda praised Zelda, as in (36) (assuming that Zelda, Oscar, and Lucie are the only relevant individuals). With She praised herSELF the speaker asserts that Zelda praised Zelda, a contradiction.

(35) Q: Whom did Zelda praise?

A: She praised no one. \# She praised herSELFF.

(36) $[[$ Whom did Zelda praise?] $=\{$ Zelda praised Zelda, Zelda praised Oscar, Zelda praised Lucie\}

Note that exactly the same reasoning should lead to a contradiction in the case of the subject $w h$-question in (37). No contradiction arises, however. This can be so if the proposition that Zelda praised Zelda is not in [[Who praised Zelda?], 
which I call a disjoint reference effect. ${ }^{12}$ If so, by asserting that Zelda praised no one, Speaker A does not assert that Zelda did not praise Zelda ${ }^{13}$ and can proceed to assert that this is the case without contradiction.

(37) Q: Who praised Zelda?

A: No one praised her. She praised herSELFF.

(38) $[[$ Who praised Zelda?] $=\{$ Oscar praised Zelda, Lucie praised Zelda $\}$

\section{A Current Question based theory of focus}

\subsection{Subject alternatives and the Question Rule}

We can now go back to the QA-pairs in (2) and (3), repeated here as (39) and (40). As discussed in section 2.1, QA-pairs are subject to the Focus Principle, which is satisfied in (39) and (40) if reflexive anaphors are arity reducers, as suggested in (4a) (Spathas 2010, 2011).

(39) Q: Who did Zelda praise?

A: She praised herSELFF.

(40) Q: Who praised Zelda?

A: ? She praised herSELFF.

A second requirement is that the answer is a partial or complete answer to the question (cf. Groenendijk and Stokhof 1984). Assuming a Hamblin/Kartunnenstyle semantics for questions, partial and complete answers can be defined as follows (from Roberts 1996, Beaver and Clark 2008).

12 The most famous disjoint reference effects are Principle B effects as in *Zelda praised $_{\text {her }}$. Baker, Johnson, and Roberts 1989 reports disjoint reference effects for passives; the implicit argument cannot be coreferential with the theme-subject in examples like They were admired. As Daniel Büring (p.c.) points out, Kehler and Büring 2008 proposes a generalized principle of disjointness in $w h$-questions containing pronouns. BBBD in (ii) captures, e.g., the disjointness effect between John and the bound pronoun in (iii). See Schlenker 2005 for a more general account of disjoint reference. As far as I have been able to determine, so far none of the existing accounts can explain the subject-object asymmetries discussed in the main text and I cannot offer a satisfying explanation either. It is important to add that by using the term 'disjoint reference effects' I do not wish to claim that all the cases mentioned here will necessarily have a unified explanation.

(ii) Be Bound or Be Disjoint (BBBD)

If a pronoun $p$ is free in the c-command domain of a (non-wh) DP $a, p$ bears a presupposition of disjointness with $a$.

(iii) $\mathrm{Who}_{1}$ thinks that John loves his ${ }_{1}$ wife?

${ }^{13}$ At least under the reasonable assumption that no one and the wh-element in the question share the same contextual restriction. 
(41) A complete answer is an utterance expressing a proposition which determines the truth-value of every proposition in [[Q]].

(42) A partial answer is an utterance which expresses a proposition which is incompatible with one or more alternative in [[Q]] and compatible with more than one logically independent alternative.

Given exhaustification, the answer in both (39) and (40), which denotes the proposition that Zelda praised Zelda is a complete answer. In the case of (39), the answer determines that it is true that Zelda praised Zelda and false that Oscar praised Zelda and Lucie praised Zelda. ${ }^{14}$ In the case of (40), the answer determines that both propositions in [[(39Q)]], as in (38), are false. The difference between (39) and (40), then, is that, given the disjoint reference effect associated with subject $w h$-question, in the case of (40) none of the propositions in [[(40Q)]] turn out to be true.

This is relevant for what Beaver and Clark (2008: 37) propose to be a third requirement for QA-congruence, namely that a question should offer at least one true alternative, as in (42) (cf. Geurts and van der Sandt 2004).

\section{(43) Current Question Rule}

The current question must contain at least one true alternative, and contain multiple alternatives which are not resolved as true or false in the common ground.

The QA-pair in (40), then, but not the one in (39), violates (43). I propose that this violation is the reason for the reduced accessibility of SA in (40). The result of the violation is a mismatch between the assumptions of the interlocutors; whereas the question does not consider the proposition that Zelda praised Zelda to be among the possible true alternatives, the answer asserts that it is in fact true. Notice that the violation in (40) does not lead to a complete break-down of communication; speaker A cannot be expected to concur with all the assumptions of speaker Q. What is expected, however, is that she explicitly rejects a question if she knows that it violates (43). I assume that this is what is done in, e.g., (37); by the use of No one praised her, Speaker A explicitly rejects Who praised Zelda? as a legitimate CQ, so that the discourse proceeds unhindered in this case.

\footnotetext{
${ }^{14}$ I take the exhaustification inference to be a scalar implicature derived by Grice's Maxim of Quantity. One could also use a grammatical theory of exhaustification as in Chierchia 2004, Fox 2006, as long as the Exh operator is treated as a free-, not a conventionally-associating operator (cf. the discussion in section 5).
} 
Reflexive anaphors and association with focus

\subsection{Deriving Generalization One}

The reasoning above explains the asymmetry between the QA-pairs in (39) and (40), but Generalization One states that the asymmetry between OA and SA holds across the board. The behaviour can be generalized to both free focus and association with focus, if we adopt a question-based discourse theory. I adopt and present here briefly Beaver and Clark's 2008 re-interpretation of the model of Roberts 1996. As in Stalnaker 1972, the objective of information-seeking discourses is taken to be to narrow down the context set, i.e. answer the question 'What's the world like?'. The main innovation is the assumption that interlocutors devise strategies to that effect using sequences of questions that stand in entailment relations (cf. Groenendijk and Stokhof 1984). So, in (44) (where indentations indicate entailment relations), answering the super-question 'What's the world like?' requires answering all the questions that it entails.

(44) What's the world like?

What happened at the meeting?

Who praised whom?

Who praised Zelda?

Whom did Zelda praise?

At any given moment in discourse, interlocutors have two legitimate moves: they can either answer the Current Question, defined in (45), or ask a question from the sequence (which is then promoted as the Current Question). The legitimacy of a discourse move is regulated by the two principles in (46) and (47) (repeated from (6)) (from Beaver and Clark 2008: 37). ${ }^{15}$

(45) Current Question (CQ)

The question mutually accepted by the interlocutors as the most immediate goal of the discourse becomes the Current Question.

(46) Discourse Principle

Utterances should be maximally relevant to the CQ.

(47) Focus Principle

[Q] is a subset of the Focus Semantic Value of (some part of) A.

According to the Discourse Principle, a declarative utterance is always an answer to the Current Question. When the CQ is not explicit, it will be accommodated. Given the Focus Principle, accommodation is driven by the focus structure of the declarative sentence, i.e. by the Focus Principle. Consider, e.g., the corrections repeated in (48) and (49). The focus structure [Zelda praised herself $f_{F}$ allows accommodation of both Whom did Zelda praise? and Who praised Zelda? . Due to

\footnotetext{
${ }^{15}$ Notice that if all declarative utterances are answers to the Current Question, Match in (5) can be dispensed with.
} 
the Discourse Principle, when a native speaker informer is confronted with a minimal discourse as in, e.g., (48) and is asked to judge its felicity, she first tries to establish the CQ speakers A and B are answering. Assuming that a correction rejects a proposed answer to the CQ (see the discussion in the next section), the native speaker uses both assertions in (48A) and (48B) in order to establish the $\mathrm{CQ}$. Of the two questions the focus structure of (48B) is compatible with, only the object $w h$-question is compatible with Speaker A's assertion (i.e. Zelda praised Oscar can only be an answer to the object $w h$-question). A native speaker, then, accommodates the question Whom did Zelda praise?. In the case of (49), exactly parallel reasoning leads a native speaker to accommodate the subject wh-question. In this case, however, accommodation of Who praised Zelda? leads to a violation of the Current Question Rule.

(48) A: Zelda praised Oscar.

B: No, she praised herSELFF.

(49) A: Oscar praised Zelda.

B: ? No, she praised herSELFF.

Turning to association with focus cases, consider again the examples involving negation, repeated here in (50), (51), and (52). According to the Focus Principle, the focus semantic value of a part of Zelda didn't praise herSELFF should be a superset of the meaning of the CQ. This could be the form without negation, i.e. Zelda praised herSELFF. As above, this allows accommodation of two CQs. In (50), the presence of She praised Oscar forces accommodation of Whom did Zelda praise?. In (51), the presence of Oscar praised her forces accommodation of Who praised Zelda? . Notice that in this case the Current Question Rule is not violated, since the proposition that Oscar praised Zelda, which is part of the meaning of Who praised Zelda?, is asserted to be true. I propose that the difficulty arises because a native speaker confronted with (51) needs to assume that the utterer of (51) considers the proposition that Zelda praised Zelda a possible answer to the question Who praised Zelda? even though this proposition is not part of the meaning of the question. In other words, if the utterer of (51) wants to answer the question Who praised Zelda? why does he bother rejecting the proposition that Zelda praised Zelda when this proposition is not a possible answer to begin with? Again, since the proposition that Zelda praised Zelda is in fact a complete answer to this question, accommodating this question is not impossible and it can happen when the continuation requires it. When confronted with an out-of-the-blue example as in (52), however, speakers will always assume that the prejacent is an answer to the question Whom did Zelda praise? for which no such issues arise. 
(51) Zelda didn't praise herSELFF. ? Oscar praised her.

(52) Zelda didn't praise herSELFF.

\subsection{Too many alternatives?}

This section presents additional evidence in favour of the Beaver and Clark/Roberts model. The observation is that narrow focus on a reflexive anaphor always generates a set of alternatives that includes either SA or OA, but never both. This is illustrated here with a case of free focus, corrective contexts. Consider, first, the correction in (53) (based on Schwarzschild's 1999 example (50)); (53B) is taken to reject only that Zelda praised Oscar, not that Oscar praised Zelda, or both that Zelda praised Oscar and Oscar praised Zelda. ${ }^{16}$ Why is that? Schwarzschild's intuition for similar examples is that focus feeds the rhetorical relations between utterances in discourse. We could assume, then, that the propositions in Speaker A's utterance that are rejected are those that are elements in the Focus Semantic Value of Speaker B's utterance (i.e. the propositions that act as antecedents for focus licensing). In the case of (53), narrow focus on Helen generates alternatives of the form Zelda praised $x$, and of the propositions in Speaker A's utterance only the proposition that Zelda praised Oscar is in that set.

(53) A: Zelda praised Oscar and Oscar praised Zelda.

B: No, Zelda praised HELENF.

The intuition fails, however, for parallel cases with reflexive anaphors, as in (54). (54B) is taken to reject that Zelda praised Oscar, and not that Oscar praised Zelda or that Zelda praised Oscar and Oscar praised Zelda. ${ }^{17}$ The simple account above predicts that both propositions are rejected, since both are in the FSV of Zelda praised herSELFF. An alternative way to explain the facts is to assume that the rejection is influenced by the choice of accommodated CQ. On the basis of (54B) one can accommodate either Whom did Zelda praise? or Who praised Zelda?. If the former is accommodated, Zelda praised herself is proffered as the only true answer to that question, so the proposition that Zelda praised Oscar is rejected. Similarly, accommodating Who praised Zelda? would lead to rejection of the proposition that Oscar praised Zelda. The question Who praised Zelda?, on the other hand, is not accommodated since it leads to a violation of the Current Question Rule.

\footnotetext{
16 The rejection of both propositions in $(52 \mathrm{~A})$ requires the use of double focus, as in ZELDA

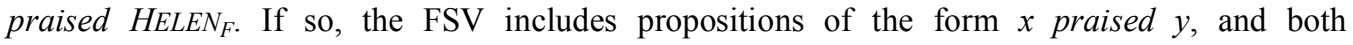
propositions in $(53 \mathrm{~A})$ are elements of this set.

${ }^{17} \mathrm{As}$ in (53), rejection of both propositions in (53A) requires the use of double focus, as in ZELDAF praised herSELFF. Double focus makes possible the accommodation of the CQ Who praised who?.
} 
(54) A: Zelda praised Oscar and Oscar praised Zelda.

B: No, Zelda praised herSELFF.

\section{An intermediate theory of association with focus}

Beaver and Clark 2008 argues for a specific analysis of conventional association with focus, according to which conventional association manifests a grammatical dependency on the Current Question. I argue that this analysis explains Generalization Two, i.e. the fact that SA do not arise with a certain class of FAOs.

\subsection{Degrees of association}

As mentioned in the introduction, Rooth 1992 distinguishes between three possible theories of focus sensitivity: (i) weak theories, (ii) strong theories, and (iii) intermediate theories. I illustrate each theory using the exclusive only, following the scalar account of the exclusive particle of Beaver and Clark 2008 in (55) (the entry is adapted from Coppock and Beaver 2011). As usual, only expresses quantification over a set of propositions C. Moreover, it comments on the relative strength of the different propositions in C. Its presuppositional component requires that no proposition in $\mathrm{C}$ is weaker or unranked with respect to the prejacent of only (i.e. the proposition expressed by the LF minus only). This is achieved through the use of MIN, as defined in (56a) ( $\geq$ represents the strength ranking, which in the cases dealt with here is based on entailment). The ordinary semantic component of only requires that no proposition in $\mathrm{C}$ is stronger than the prejacent. This is achieved through the use of MAX, as defined in (56b).

(55) $[[$ only $]]=\lambda p \cdot \operatorname{MIN}(p) \cdot \operatorname{MAX}(p)$

(56) a. $\operatorname{MIN}(\mathrm{p})=\exists \mathrm{q} \in \mathrm{C}[\operatorname{true}(\mathrm{q}) \wedge \mathrm{q} \geq \mathrm{p}]$

b. $\operatorname{MAX}(\mathrm{p})=\forall \mathrm{q} \in \mathrm{C}[\operatorname{true}(\mathrm{q}) \rightarrow \mathrm{p} \geq \mathrm{q}]$

The question, then, is how the value of the variable $\mathrm{C}$ is determined and why it is dependent on focus placement. In a strong theory, $\mathrm{C}$ is contextually resolved. Pragmatic principles, like, e.g., the Discourse Principle in (46), determine that, in most cases, it is resolved to the same object that acts as the antecedent for focus licensing. In the Roberts/Beaver and Clark model, where every declarative sentence is an answer to the CQ, the relevant object is the CQ. Association with focus, then, is only indirect, via, e.g., the CQ. In a weak theory, association with focus is written directly in the semantics of only. In, e.g., Rooth 1985, the quantifier ranges over the propositions in the sentences alternative value. MIN and MAX, then, could be defined as in (57). 


$$
\begin{aligned}
& \text { a. } \operatorname{MIN}(p)=\exists q \in[[p]]^{f}[\operatorname{true}(q) \wedge q \geq p] \\
& \text { b. } \operatorname{MAX}(p)=\forall q \in[[p]]^{f}[\operatorname{true}(q) \rightarrow p \geq q]
\end{aligned}
$$

Although strong theories derive association with focus for only, they leave open the possibility that the truth-conditional content will not always be affected by the placement of focus; although the free variable can, and generally will, be resolved to the denotation of the CQ, it does not have to be. The existence or nonexistence of such cases has been the main empirical contention between defenders of weak and strong theories. Beaver and Clark 2008 provides a variety of tests that distinguish between weak and strong association with focus. Crucially, they show that not all FAOs behave alike, and that a typology of FAOs should include the three categories mentioned above; QAOs, FrAOs, and CAOs. Among those, only CAOs exhibit a conventionalized dependency. Crucially, the conventionalized dependency is not a dependency with the FSV, but a dependency with the Current Question, as in (58). The discourse function of CAOs is to comment, in one way or another, on the CQ. For example, the exclusive only comments on the relative strength of possible answers to the CQ.

$$
\begin{aligned}
& \text { a. } \operatorname{MIN}(p)=\exists \mathrm{q} \in \mathrm{CQ}[\operatorname{true}(\mathrm{q}) \wedge \mathrm{q} \geq \mathrm{p}] \\
& \text { b. } \operatorname{MAX}(\mathrm{p})=\forall \mathrm{q} \in \operatorname{CQ}[\operatorname{true}(\mathrm{q}) \rightarrow \mathrm{p} \geq \mathrm{q}]
\end{aligned}
$$

\subsection{Deriving Generalization Two}

Consider now the contrast between always and only in licensing SA, repeated here in (59) and (60). Always is a universal quantifier that ranges over a free variable, a variable over sets of events (von Fintel 1994). Since always is a FrAO according to Beaver and Clark 2008, the relevant reading in (59) will come about if the variable is resolved to a set of events of the type $x$ praised Zelda in e. As long as the context makes such a set available, SA is possible. In the case of (60), SA will arise if only ranges over a set of propositions of the type $x$ praised Zelda. The crucial difference with (59) is that the set can only be picked up through the CQ Who praised Zelda?. Given the focus structure in (60), this CQ can be accommodated. It exhibits, however, a disjoint reference effect, so that the relevant set will include, e.g., the proposition that Oscar praised Zelda, but not the proposition that Zelda praised Zelda. Recall that the presupposition of only requires that there is at least one true proposition in $\llbracket C Q \rrbracket$ that is equal or stronger than Zelda praised Zelda. Since the proposition that Zelda praised Zelda is not in $\llbracket C Q \rrbracket$, there is no proposition in $\llbracket \mathrm{CQ} \rrbracket$ to satisfy the presupposition of only. The deviance of SA in (60), then, is the result of presupposition failure.

(59) Zelda always praises herSELFF. ? No one else ever praises her.

(60) Zelda only praised herSELFF. \# No one else praised her. 
More generally, given the discourse function of CAOs as providing comments on the CQ and the fact that the CQ is part of their conventional meaning, subject whquestions will always lead to violations of the Current Question Rule, but, crucially, for one and the same speaker. Whereas in examples like (2) speaker A gives an answer that does not conform to the assumptions of speaker Q, in, e.g., (60) the speaker must himself accept the CQ together with all its assumptions, as the CQ is part of the meaning of only. The speaker, then, puts forward a CQ (via accommodation) according to which the proposition that Zelda praised Zelda is not a possible answer, and answers the question by asserting that Zelda praised Zelda, which is clearly a contradiction. It is predicted, then, that SA will not improve if the CQ is explicit, as in (61A1), or even if it is explicitly rejected, as in (61A2), since it is immediately re-introduced through the lexical semantics of only.

(61) Q: Who praised Zelda?

A1: \# She only praised herSELFF.

A2: No one praised her. ${ }^{\#}$ She only praised herSELFF.

\section{Conclusions}

Recent literature on focus theory has unearthed several differences between focus associating operators and has made the first steps into establishing a typology of such elements. This paper adds to this line of research by establishing a further environment that distinguishes between free and conventional association with focus: the licensing of Subject Alternatives generated by narrowly focusing reflexive anaphors in English. Moreover, it is argued that these differences can be explained if one adopts a theory of focus that is based on a specific type of discourse model, one built around the notion of Current Question.

The analysis presented here leaves open several issues and opens up possible avenues for further research. The analysis is based on the existence of asymmetries with regard to disjoint reference effects between subject and object wh-questions, for which no account was offered. As mentioned in footnote 10, Subject Alternatives do seem to arise with a certain class of predicates, a fact attributed to the availability of a construal in which herself is an anti-assistive intensifier. If so, there exists a second road to SA which is based on intensification rather than reflexivization and is not subject to the restrictions of Generalization Two. If this suggestion is on the right track, the availability of SA with Conventionally Associating Operators becomes a way to probe into the semantics and the composition of complex reflexive anaphors across languages answering some long-standing questions within Binding Theory. 
Reflexive anaphors and association with focus

\section{References}

Ahn, Byron. 2012. Twin reflexives. Invited talk, January 20, 2012. University of Arizona Linguistics Colloquium.

Baker, Mark, Kyle Johnson, and Ian Roberts. 1989. Passive arguments raised. Linguistic Inquiry 20, 219-51.

Beaver, David and Brady Clark. 2008. Sense and Sensitivity. Malden, MA: WileyBlackwell.

Bonomi, Andrea and Paolo Casalegno. 1993. Only: association with focus in event semantics. Natural Language Semantics 2, 1-45.

Büring, Daniel. 2008 What's New (and what's Given) in the theory of focus?. Ms. Berkeley Linguistic Society.

Chierchia, Gennaro. 2004. Scalar implicatures, polarity phenomena, and the syntax/pragmatics interface. In Andriana Belletti (ed.), Structures and Beyond: The Cartography of Syntactic Structures. New York: Oxford University Press.

von Fintel, Kai. 1994. Restrictions on quantifier domains. PhD Dissertation. Amherst: University of Massachusetts.

Coppock, Elizabeth and David Beaver. 2011. Sole sisters In Neil Ashton, Anca Chereches, and David Lutz (eds.), Semantics and Linguistic Theory (SALT) 21, 197-217. Ithaca, NY: CLC.

Fox, Danny. 2006. Free choice and the theory of scalar implicatures. Ms. MIT.

Geurts, Bart and Rob van der Sandt. 2004. Interpreting focus. Theoretical Linguistics 30, 1-44

Groendijk, Jeroen and Martin Stokhof. 1984. Studies on the Semantics of Questions and the Pragmatics of Answers. Amsterdam: University of Amsterdam.

Jackendoff, Ray. 1972. Semantic Interpretation in Generative Grammar. Cambridge, Mass: MIT Press.

Kehler, Andrew and Daniel Büring. 2008. Be Bound or Be Disjoint!. NorthEastern Linguistic Society (NELS) 38. Ottawa

Krifka, Manfred. 1992. A compositional semantics for multiple focus constructions. In Joachim Jacobs (ed.), Informationsstruktur und Grammatik, 17-53. Opladen: Westdeutscher Verlag.

Krifka, Manfred. 1995. Focus and the interpretation of generic sentences. In Manfred Krifka, Francis Jeffry Pelletier, Greg Carlson, Gennaro Chierchia, Godehard Link, and Alice ter Meulen (eds.), The Generic Book, 238-264. The University of Chicago Press.

Roberts, Craige. 1996. Information structure in discourse: towards an integrated formal theory of pragmatics. OSU Working Papers in Linguistics 49, 91-136. Papers in Semantics. The Ohio State University. 
Roberts, Craige. 2004. Context in dynamic interpretation. In Laurence H. Horn and Gregory Ward (eds.), Handbook of Contemporary Pragmatic Theory, 197-220. Malden, MA: Blackwell Publishing.

Rooth, Mats. 1985. Association with Focus. PhD Dissertation. University of Massachusetts at Amherst.

Rooth, Mats. 1992. A theory of Focus interpretation. Natural Language Semantics $1,75-116$.

Schlenker, Philippe. 2005. Non-redundancy: towards a semantic reinterpretation of binding theory. Natural Language Semantics 13, 1-92.

Schwarzschild, Roger. 1999. GIVENness, AvoidF and other constraints on the placement of accent. Natural Language Semantics 7, 141-77.

Spathas, Giorgos. 2010. Focus on anaphora. PhD Dissertation. LOT dissertation series.

Spathas, Giorgos. 2011. Focus on reflexive anaphors. In Neil Ashton, Anca Chereches, and David Lutz (eds.), Semantics and Linguistic Theory (SALT) 21, 471-488. Ithaca, NY: CLC.

Spathas 2012. Reflexivizers and intensifiers: consequences for a theory of focus. Sinn und Bedeutung 17, 581-598.

Stalnaker, Robert. 1972. Pragmatics. In Donald Davidson and Gilbert Hartman (eds.), Semantics of Natural Language, 380-397. Reidel: Dordrecht.

Stephens, Nola. 2006. Agentivity and the virtual reflexive construction. In Benjamin Lyngfelt and Torgrim Solstad (eds.), Demoting the agent: Passive, middle and other voice phenomena, 275-300. Amsterdam: John Benjamins.

Truckenbrodt, Hubert. 1995. Phonological phrases: their relation to syntax, focus, and prominence. PhD Dissertation. MIT.

von Stechow, Arnim. 1990 Focusing and backgrounding operators. In Werner Abraham (ed.), Discourse Particles, 37-84. Amsterdam: John Benjamins.

Wagner, Michael. 2005. Prosody and recursion. PhD Dissertation. MIT.

Giorgos Spathas

Institut für Linguistik: Anglistik

Keplerstr. 17

Stuttgart, 70174

Germany

g.spathas@gmail.com / giorgos.spathas@,ifla.uni-stuttgart.de 